\title{
Outcomes of Laparoscopic Liver Resection for Patients with Multiple Hepatocellular Carcinomas Meeting the Milan Criteria: A Propensity Score-Matched Analysis
}

\author{
Yufu Peng, MD, ${ }^{*}$ Fei Liu, MD, ${ }^{*}$ Hongwei Xu, MD, Xiang Lan, MD, \\ Yonggang Wei, MD, and Bo Li, MD, PhD
}

\begin{abstract}
Background: Until now, there is little knowledge about the value of laparoscopic liver resection (LLR) for patients with multiple hepatocellular carcinomas (HCC). This study was performed to assess the efficacy and outcomes of LLR versus open liver resection (OLR) for patients with multiple HCC meeting the Milan criteria. Methods: One hundred fifteen patients with multiple HCC meeting the Milan criteria who underwent liver resection from April 2015 to March 2018 were enrolled into this study. According to the different surgical procedures, patients were divided into LLR group and OLR group. Perioperative and oncological outcomes were compared between the two groups after propensity score matching (PSM) with 1:1 match.

Results: Thirty three patients were included into each group after PSM with well-balanced basic level. The intraoperative blood loss in LLR group was less than OLR group before PSM (median, 200 vs. $300 \mathrm{~mL}$, $P=.004$ ), but the difference was not statistically significant after PSM (median, 200 vs. $300 \mathrm{~mL}, P=.064)$. LLR group showed shorter postoperative hospital stay when compared with OLR group (median, 7 vs. 8 days, respectively, $P=.014$ ). The perioperative complications and early mortality were comparable in both groups. There were no significant differences in the term of overall survival (OS. $P=.502)$ or recurrence-free survival (RFS. $P=.887$ ) between the two groups after PSM.

Conclusions: LLR could be safely and feasibly performed for patients with multiple HCC meeting the Milan criteria in selected patients. It does not increase the risks of postoperative complications and has a similar oncological outcomes compared to OLR.
\end{abstract}

Keywords: laparoscopic liver resection, multiple hepatocellular carcinomas, Milan criteria, complications, survival rate

\section{Introduction}

$\mathbf{L}$ IVER TRANSPLANTATION is the most effective treatment for patients with multiple hepatocellular carcinomas (HCC) meeting the Milan criteria, but hepatic resection is still selected as first-line treatment due to the limitation of organ shortages. ${ }^{1,2}$ However, patients with multiple HCC have unique characteristics, including major resection extent more likely to be performed, $\mathrm{R} 0$ resection more difficult to be achieved, require multiple resections in different segments, and poor long-term outcomes. For these patients, some trials have indicated that liver resection may offer significantly better oncological outcomes than other treatments, such as radiofrequency ablation (RFA), transarterial chemoembolization (TACE), and so on. ${ }^{3-5}$ According to a review reported by Chow and colleagues ${ }^{1}$ and many related trials, ${ }^{3-7}$ open

Department of Liver Surgery, Center of Liver Transplantation, West China Hospital of Sichuan University, Chengdu, China.

*These authors contributed equally to this work.

(C) Yufu Peng et al. 2019; Published by Mary Ann Liebert, Inc. This Open Access article is distributed under the terms of the Creative Commons Attribution Noncommercial License (http://creativecommons.org/licenses/by-nc/4.0/) which permits any noncommercial use, distribution, and reproduction in any medium, provided the original author(s) and the source are cited. 
liver resection (OLR) as the potentially curative therapy is widely adopted for patients with multiple HCC, and it remains the standard treatment for these patients.

Laparoscopic liver resection (LLR) has been widely performed following improvements of surgical instruments and surgeons' experience since the First International Consensus Conference on Laparoscopic Liver Surgery Convened in Louisville in 2008. ${ }^{8}$ The indications have been expanded, beginning with local resections and evolving to major resections, posterior-superior resections, consolidated cirrhosis, and anatomic resections. ${ }^{9-11}$ The diseases of patients under LLR were selected from benign to malignancy. As being a minimally invasive surgery, LLR was reported with advantages compared with traditional operation, including less intraoperative blood loss, fewer complications, shorter hospital stays, and equivalent long-term outcomes. ${ }^{12,13}$ Even many studies have been carried out to evaluate the outcomes of LLR in terms of age, body mass index (BMI), surgical position, surgical approaches, location of the tumor, size of the tumor, contact with major vessel, previously abdominal surgery history, liver cirrhosis, resection extent, surgical instrument and techniques, and learning curve..$^{9-11,14-17}$

Although a study had evaluated the safety of LLR for patients with multiple HCC, ${ }^{18}$ there were some limitations in it. First, the size of sample was too small. Second, it was performed to assess the feasibility of laparoscopic approach for multiple $\mathrm{HCC}$ compared with a single $\mathrm{HCC}$, and patients under laparoscopic RFA were also enrolled into the study. Last but not least, it did not calculate the outcomes of LLR for multiple HCC compared to OLR, especially for the long-term outcomes. However, OLR as the standard approach is widely used for patients with multiple HCC, but there is little knowledge about the value of LLR for these patients compared with OLR. In the previous published studies about the outcomes of LLR versus OLR, the number of tumors was served as a variety of the baseline characteristics, whereas patients with solitary tumor were also mixed in these studies.

Therefore, the purpose of this study was performed to analyze the perioperative and oncological outcomes of LLR for patients with multiple HCC meeting the Milan criteria compared to OLR.

\section{Materials and Methods}

\section{Study design}

Between April 2015 and March 2018, all patients with multiple HCC who received curative hepatectomy in our center were retrospectively collected from a prospectively established database. The inclusion criteria were as follows: (1) male or female patients aged 18-75 years; (2) with ChildPugh A or B hepatic function; (3) histopathological confirmation of HCC; and (4) multiple HCC within Milan criteria: the number of HCC was 2 or 3 and the diameter of the biggest tumor $\leq 3 \mathrm{~cm}$, without the presence of extrahepatic metastasis, or tumor thrombus in portal vein or other major vascular. The exclusion criteria were as follows: (1) liver resection combined with other abdominal organ resection except for gallbladder; (2) the surgical procedures combined resection and RFA; and (3) repeated liver resection.

According to the different surgical procedures, patients were divided into LLR group and OLR group. The selection between the LLR and OLR was depended on the consultation of patients and surgeons. After surgeons fully informed patients about the pros and cons of LLR versus OLR, the final decision was made by the patients. Preoperative evaluations included complete blood count, liver function, and tumor markers, triphasic computed tomography (CT), or magnetic resonance imaging (MRI). To prevent the omissions of tumors, contrast-enhanced ultrasound was routinely performed in preoperative evaluations. This study was undertaken in accordance with the principles of the Declaration of Helsinki and was approved by the clinic trial Ethics Committee of West China hospital.

\section{Surgical procedures}

The detailed techniques for laparoscopic liver surgery have been described in our previous studies. ${ }^{19,20}$ In general, all patients were tilted into left lateral decubitus position followed by intravenous total anesthesia. The operator stood on the patient's right side, and the assistant and scopist were positioned on the left side. Pneumoperitoneum was established by carbon dioxide and maintained at $12-14 \mathrm{mmHg}$. A 12-mm port was used for laparoscope, whereas two 12-mm ports and 5-mm ports were applied for operation. Intraoperative ultrasonography was routinely used to confirm the positions of tumors, prevent the omissions of small tumors, and guide the transection line. The superficial liver parenchyma was transected by harmonic scalpel (Ethicon EndoSurgery), whereas the deeper parenchyma transaction was completed with a combination of harmonic scalpel and laparoscopic cavitron ultrasonic surgical aspirator $\left(\right.$ CUSA $^{\circledR}$; Valleylab, Inc.) or Ligasure $^{\mathrm{TM}}$ (Valleylab, Inc.). Small branches of Glisson's pedicles and hepatic vein $(\geq 5 \mathrm{~mm})$ were ligated by Hem-o-lok ${ }^{\circledR}$ or titanium clips, whereas the main Glisson's pedicle or hepatic vein were all dissected by the laparoscopic linear stapler(Endopath ${ }^{\circledR}$ Endocutter; Ethicon Endo-surgery). The resected specimens were placed into a specimen bag and removed through a suprapubic incision or an upper abdominal midline incision. After hemostasis and irrigation of the resected liver surface, drainage tube was placed near the surgical bed.

For open procedure, a reverse L-incision was conducted with patients under intravenous total anesthesia. The operating procedure was similar to LLR, but the clamp crushing or CUSA was performed as the main methods for liver parenchyma dissection.

For all patients, local resection or anatomical resection was performed according to the extent of the tumor. Anatomical resection was mainly achieved using the Glissonian pedicle approach as previously reported. ${ }^{21}$ Pringle maneuver and the central venous pressure within $5 \mathrm{mmHg}$ were applied to control the blood loss in both groups. The Pringle's maneuver technique of LLR had been described in detail in our previous study. ${ }^{19}$

\section{Postoperative management and follow-up}

Postoperative evaluations, including complete blood count and liver function tests were usually conducted on postoperative days $1,3,5$, and 7 . An abdominal ultrasonography was routinely conducted before outpatient to detect whether the omissions of small tumors exist.

All patients were followed after discharge, and the follow-up was carried out at 1 month after surgery, quarterly in the first year, and every 3-6 month thereafter. Routine investigations 
included complete blood count, hepatic function, tumor markers, and abdominal ultrasonography, even CT or MRI if necessary.

\section{Definition}

The Milan criteria was defined as a single tumor $\leq 5 \mathrm{~cm}$ or no more than 3 tumors with the biggest tumor $\leq 3 \mathrm{~cm}$ in patients without extrahepatic metastasis and tumor thrombus in major vascular. ${ }^{22}$ Major complication was identified as Clavien-Dindo grade III and above. ${ }^{23}$ Liver failure was defined according to the "50-50" criterion. ${ }^{24}$ Ascites was defined as postoperative abdominal daily drainage fluid $>10 \mathrm{~mL} / \mathrm{kg}$ of body weight. ${ }^{25}$ Hemorrhage was determined according to the drop of postoperative hemoglobin level $>3 \mathrm{~g} / \mathrm{dL}$ compared with postoperative basic level. ${ }^{26}$ Biliary leakage was identified as the concentration of bilirubin in the abdominal drainage $\geq 3$ times than that in serum after post-

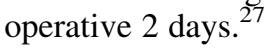

\section{Statistical analysis}

A propensity score-matched (PSM) analysis was used to limit the selection biases and reduce confusion of the retrospective study. The propensity score was estimated using logistical regression and enrolled following factors: age, BMI, American Society of Anesthesiologists (ASA), comorbidities, prior TACE, prior RFA, previously abdominal surgery history, alpha fetoprotein, cirrhosis, tumor number, the biggest tumor diameter, total tumor diameter, distribution (one lobe vs. bi-lobe), and resection extent (major vs. minor). PSM was conducted by using a 1:1 ratio based on the nearest neighbor matching method without replacement.

Before PSM, continuous variables were compared by Mann-Whitney U test and categorical data were compared by the Chi-square test or 1-tailed Fisher's exact test. After PSM, continuous variables were compared using the Wilcoxon signed-rank test and categorical data were compared using the McNemar's test. Survival outcomes were calculated by using Kaplan-Meier method and compared with the log-rank test.

$P$-value $<.05$ were regarded as significant. All statistical analyses above were conducted with SPSS ${ }^{\circledR}$ software version 22.0 (IBM Corp. Released 2013. IBM SPSS Statistics for Windows, Version 22.0. Armonk, NY: IBM Corp.).

\section{Results}

Patients

Between April 2015 and March 2018, 136 patients with multiple HCC within the Milan criteria underwent liver hepatectomy were identified in our center. Twenty one patients were excluded for the surgical procedures that combined liver resection and RFA. Eventually, 115 patients satisfied the criteria and were eligible for analysis; of which, 35 patients were in the LLR and 80 patients were in the OLR according to the different surgical procedures. After PSM, 33 patients were in each group, respectively.

\section{Baseline characteristics}

The preoperative basic characteristics are shown in the Table 1. Before PSM, the distribution of tumors was statis- tically different between the two groups $(P=.019)$. After PSM, the basic levels of two groups were well matched.

\section{Surgical outcomes}

LLR group showed less intraoperative bleeding compared to OLR group before PSM (median, 200 vs. $300 \mathrm{~mL}$, $P=.004$ ), but the difference was not statistically significant after PSM (median, 200 vs. $300 \mathrm{~mL}, P=.064$ ). Operative time, blood transfusion, resection extent, and R0 resection between the two groups were comparable both before PSM and after PSM. One case was converted to open surgery because of the unclear field exposure (Table 2).

\section{Postoperative outcomes}

Overall complications, major complications (ClavienDindo grade $\geq 3$ ), liver-specific complications (liver failure and biliary leakage, except for ascites), surgical-related complications (hemorrhage and wound infection), and general complications (respiratory infection, pleural effusion, and urinary tract infection) were comparable in both groups before PSM and after PSM. Although the incidence of ascites was not significantly different in the two groups before and after PSM (0\% vs. $11.3 \%, P=.091 ; 0 \%$ vs. $15.2 \%, P=.063$, respectively), there is a tendency that ascites occurred less frequency in LLR group than OLR group. The postoperative hospital stay was significantly shorter in LLR group compared to OLR group before and after PSM (median, 7 vs. 8 days, $P=.015$; median, 7 vs. 8 days, $P=.014$, respectively). The early mortality (within 90 days after operation) was 0 in the both groups (Table 3).

\section{Long-term outcomes}

The median follow-up time was 13 months (range, 1-40 months). There were no significant differences in the term of overall survival (OS. $P=.502$ ) and recurrence-free survival (RFS. $P=.887$ ) between the two groups after PSM (Fig. 1). The 1- and 3-year OS rates were $95.8 \%$ and $77.0 \%$ in the LLR group, and were $92.8 \%$ and $77.1 \%$ in the OLR group (Fig. 1A). The 1- and 3-year RFS rates were $71.9 \%$ and $51.4 \%$ in the LLR group, and were $79.1 \%$ and $46.2 \%$ in the OLR group (Fig. 1B).

\section{Discussion}

For patients with multiple HCC meeting the Milan criteria, liver transplantation has been widely accepted as the optimal treatment. However, due to the limitation of organ shortages, liver resection is still performed as the first-line treatment. ${ }^{1,2}$ Until now, there is little knowledge about the value of LLR for patients with multiple HCC, thus the aim of this study was to assess the short-term and long-term outcomes of LLR versus OLR for these patients. According to our analysis, patients in the LLR group had less intraoperative blood loss and shorter postoperative hospital stay when compared to OLR group. Moreover, the postoperative complications, early mortality, and oncological outcomes were comparable in both groups.

In this study, we used PSM to limit the selection biases and reduce confusion of the retrospective study. Before PSM, rates of tumors located in both liver lobes in the LLR group were significantly higher than that in the OLR group. 


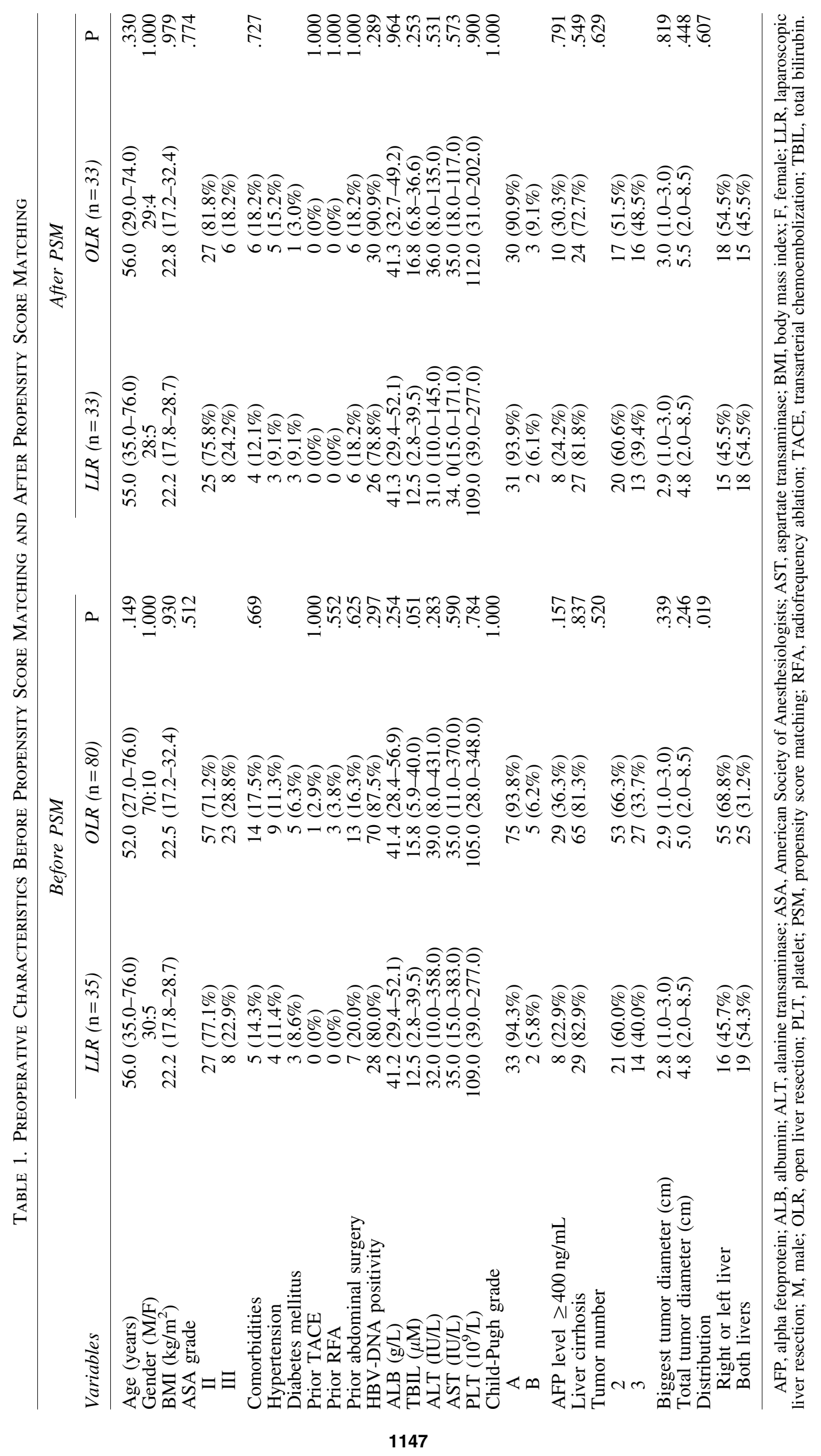


Table 2. Surgical Outcomes Before Propensity Score Matching and After Propensity Score Matching

\begin{tabular}{|c|c|c|c|c|c|c|}
\hline \multirow[b]{2}{*}{ Variables } & \multicolumn{3}{|c|}{ Before PSM } & \multicolumn{3}{|c|}{ After PSM } \\
\hline & $\operatorname{LLR}(\mathrm{n}=35)$ & $O L R(\mathrm{n}=80)$ & $\mathrm{P}$ & $\operatorname{LLR}(\mathrm{n}=33)$ & $O L R(\mathrm{n}=33)$ & $\mathrm{P}$ \\
\hline Operative time (min) & $225(80-505)$ & $210(105-340)$ & .123 & $225(80-505)$ & $210(120-205)$ & .432 \\
\hline Blood loss (mL) & $200(20-1500)$ & $300(50-1200)$ & .004 & $200(20-1500)$ & $300(50-1000)$ & .064 \\
\hline Blood transfusion & $1(2.9 \%)$ & $8(10.0 \%)$ & .350 & $1(3.0 \%)$ & $4(12.1 \%)$ & .375 \\
\hline Conversion & $1(2.9 \%)$ & - & - & $1(3.0 \%)$ & - & - \\
\hline Resection extent & & & .376 & & & 1.000 \\
\hline major & $18(51.4 \%)$ & $34(42.5 \%)$ & & $17(51.5 \%)$ & $16(48.5 \%)$ & \\
\hline minor & $17(48.6 \%)$ & $46(57.5 \%)$ & & $16(48.5 \%)$ & $17(51.5 \%)$ & \\
\hline R0 resection & $35(100.0 \%)$ & $79(98.8 \%)$ & 1.000 & $33(100.0 \%)$ & $33(100.0 \%)$ & 1.000 \\
\hline Satellites present & $6(17.1 \%)$ & $15(18.8 \%)$ & .837 & $5(15.2 \%)$ & $4(12.1 \%)$ & 1.000 \\
\hline Microvascular invasion & $11(31.4 \%)$ & $21(26.3 \%)$ & .569 & $10(30.3 \%)$ & $10(30.3 \%)$ & 1.000 \\
\hline Capsular invasion & $10(28.6 \%)$ & $23(28.8 \%)$ & .984 & $9(27.3 \%)$ & $12(36.4 \%)$ & .607 \\
\hline Poor differentiation & $18(51.4 \%)$ & $31(38.8 \%)$ & .206 & $17(51.5 \%)$ & $17(51.5 \%)$ & 1.000 \\
\hline
\end{tabular}

LLR, laparoscopic liver resection; OLR, open liver resection; PSM, propensity score matching.

According to the previous study, patients with multiple tumors located in the both lobes had the poor oncological outcomes compared with whose tumors located in the left or right lobe. ${ }^{28,29}$ After PSM, the baseline characteristics of the two groups were well matched.

Intraoperative blood loss still remains the focus issue for liver surgery procedures. Our study revealed that the intraoperative bleeding in LLR group was less than that in OLR group, even though it did not reach a significant statistical difference after PSM. The result was in compliance with some previous published studies, which could be attributed that the intraoperative pneumoperitoneum, meticulous hemostasis with the image magnification, and minimally invasive of the abdominal wall caused less bleeding in the LLR group compared with the OLR group. ${ }^{9-13,30}$ In our center, Pringle maneuver was routinely performed to control the bleeding in liver resection procedure, combined with the central venous pressure within $5 \mathrm{mmHg}$, the surgical field of resected liver surface was cleaned with the less bleeding. What's more, CUSA or LigaSure was usually applied for dissection of liver parenchyma, which could further reduce the intraoperative blood loss.

In our study, the incidence of tumor omissions was 0 in both groups. The issue of tumor omission in procedure is a matter of great concern to surgeons. Especially for multiple HCC within the Milan criteria, the tumors could be left out in liver resection due to some tumors less than $1 \mathrm{~cm}$ in diameter, which lead to an early recurrence of tumors. However, some surgeons could have a concern that the tumor omissions could easily occur in LLR for the absence of touch feeling. In our center, CT, MRI, and contrast-enhanced ultrasound were routinely performed in preoperative evaluations to prevent the omissions of tumors. In addition, instead of palpation, laparoscopic ultrasonography was routinely conducted to confirm the positions of tumors and prevent the omissions of small tumors, and ICG fluorescence imaging was gradually used to help us identify the location and number of tumors in any time. Moreover, an abdominal ultrasonography was routinely conducted before discharge to detect whether the omissions of small tumors exist, and no tumor omissions

Table 3. Postoperative Outcomes Before Propensity Score Matching and After Propensity Score Matching

\begin{tabular}{|c|c|c|c|c|c|c|}
\hline \multirow[b]{2}{*}{ Variables } & \multicolumn{3}{|c|}{ Before PSM } & \multicolumn{3}{|c|}{ After PSM } \\
\hline & $\operatorname{LLR}(\mathrm{n}=35)$ & $O L R(\mathrm{n}=80)$ & $\mathrm{P}$ & $\operatorname{LLR}(\mathrm{n}=33)$ & $O L R(\mathrm{n}=33)$ & $\mathrm{P}$ \\
\hline Overall complications & $6(17.1 \%)$ & $20(25.0 \%)$ & .354 & $6(18.2 \%)$ & $7(21.2 \%)$ & 1.000 \\
\hline Major complications & $1(2.9 \%)$ & $5(6.3 \%)$ & .766 & $1(3.0 \%)$ & $1(3.0 \%)$ & 1.000 \\
\hline Liver specific complications & $4(11.4 \%)$ & $14(17.5 \%)$ & .410 & $4(12.1 \%)$ & $5(15.2 \%)$ & 1.000 \\
\hline Liver failure & $3(8.6 \%)$ & $6(7.5 \%)$ & 1.000 & $3(9.1 \%)$ & $1(3.0 \%)$ & .625 \\
\hline Ascites & $0(0 \%)$ & $9(11.3 \%)$ & .091 & $0(0 \%)$ & $5(15.2 \%)$ & .063 \\
\hline Biliary leakage & $1(2.9 \%)$ & $0(0 \%)$ & .304 & $1(3.0 \%)$ & $0(0 \%)$ & 1.000 \\
\hline Surgical related complications & $1(2.9 \%)$ & $4(5.0 \%)$ & .983 & $1(3.0 \%)$ & $0(0 \%)$ & 1.000 \\
\hline Hemorrhage & $1(2.9 \%)$ & $3(3.8 \%)$ & 1.000 & $1(3.0 \%)$ & $0(0 \%)$ & 1.000 \\
\hline Wound infection & $0(0 \%)$ & $1(1.3 \%)$ & 1.000 & $0(0 \%)$ & $0(0 \%)$ & 1.000 \\
\hline General complications & $2(5.7 \%)$ & $5(6.3 \%)$ & 1.000 & $2(6.1 \%)$ & $2(6.1 \%)$ & 1.000 \\
\hline Respiratory infection & $1(2.9 \%)$ & $3(3.8 \%)$ & 1.000 & $1(3.0 \%)$ & $1(3.0 \%)$ & 1.000 \\
\hline Pleural effusion & $1(2.9 \%)$ & $2(2.5 \%)$ & 1.000 & $1(3.0 \%)$ & $0(0 \%)$ & 1.000 \\
\hline Urinary tract infection & $0(0 \%)$ & $1(1.3 \%)$ & 1.000 & $0(0 \%)$ & $1(3.0 \%)$ & 1.000 \\
\hline Postoperative stay (days) & $7(2-12)$ & $8(5-38)$ & .015 & $7(2-12)$ & $8(5-38)$ & .014 \\
\hline Early mortality & $0(0 \%)$ & $0(0 \%)$ & 1.000 & $0(0 \%)$ & $0(0 \%)$ & 1.000 \\
\hline
\end{tabular}

LLR, laparoscopic liver resection; OLR, open liver resection; PSM, propensity score matching. 

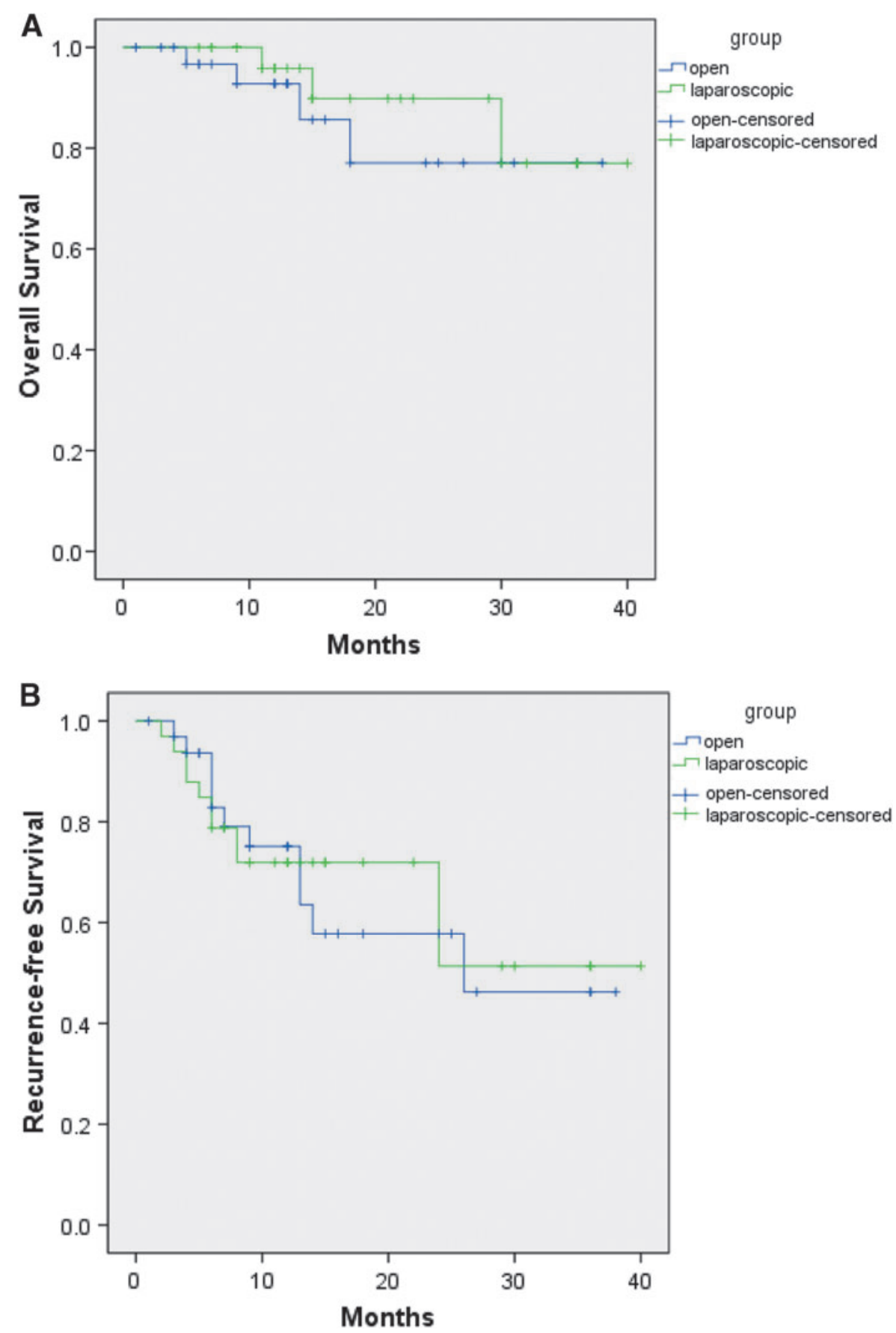

FIG. 1. (A) Overall survival $(P=.502)$ after PSM; (B) Recurrence-free survival $(P=.887)$ after PSM. PSM, propensity score matching.

were found in all the patients. These could be the reasons for tumor omissions that did not occur in the two groups in our study.

This present study showed that postoperative complications were no statistically different in the two groups, but there is a tendency that ascites occurred in LLR group less frequently than OLR group. This result was in compliance with our previous study, ${ }^{31}$ as we reported earlier, that patients with $\mathrm{HCC}$ were often accompanied with liver cirrhosis in China, which led to portal hypertension and increasing of abdominal collateral circulation. But minimally invasive of abdominal collateral circulation in LLR could decrease the occurrence of ascites, which could be the reason of our results. However, according to a meta-analysis reported by Michal Pedziwiatr et al., ${ }^{13}$ the major complications were less in LLR than in OLR, which was different from our results. Reason for this could be that the meta-analysis included patients with different baseline levels. In addition, with thousands of liver resection experience in our center, the major complication rate was relatively low. Due to the small sample size, the difference between the two groups was not obvious. 
Based on this study, our analysis demonstrated that LLR group had shorter postoperative hospital stay. The result was similar to other literature' reports, due to the less postoperative pain, early mobilization, and the early recovery of gastrointestinal function, the postoperative hospital stay in the LLR group was significantly shorter than the OLR group. ${ }^{32-34}$ With the proposition of enhanced recovery after surgery (ERAS) program, ERAS also has been conducted to patients in our department, in a broad sense, LLR as a minimally invasive surgery follows the concept of ERAS.

With regard to oncological outcomes, there remains debate about whether LLR is safe for patients with HCC compared with OLR. In our study, the long-term outcomes, including OS and RFS, were no significant difference between the LLR group and the OLR group. Up to now, most of previous published reports suggested that the survival outcomes were comparable in both groups. ${ }^{9-11,13,31}$ But the control of resection margin for multiple $\mathrm{HCC}$ is more difficult than that for isolated tumor during liver resection producers, and many articles indicated that patients with multiple tumors have a poorer long-term survival rates than those who are with a solitary tumor. ${ }^{35,36}$ Especially for LLR, some surgeons could have a concern that $\mathrm{R} 0$ resection cannot be guaranteed due to the absence of touch feeling. However, this study indicated that the $\mathrm{R} 0$ resection seems to be similar between the two groups. In our center, laparoscopic ultrasonography was routinely used to confirm the positions of tumors and guide the transection line, so that the negative resection margin was secured. What's more, pathologic variables, including poor differentiation, satellites present, capsular invasion, and microvascular invasion, which could affect the oncological outcomes, were not different in both groups. Thus, the oncological outcomes were comparable in LLR and OLR groups, which were in compliance with previous published study. 37

The small sample size of patients is the first limitation in our study, thus the potential differences of some outcomes between the two groups may not show up. Another limitation of this study is its retrospective, nonrandomized design. However, there is little knowledge about the value of LLR for patients with multiple HCC, and we try to decrease the selection bias by using the PSM. Nevertheless, even with the utilization of PSM in this comparative study, it is still associated with selection bias. Therefore, further large sample size and well-designed trials are needed.

In conclusion, this study was the first time to calculate the efficacy and outcomes of LLR versus OLR in patients with multiple HCC meeting the Milan criteria worldwide. Our analysis indicated that LLR had less intraoperative bleeding and shorter postoperative hospital stay when compared with OLR, and the $\mathrm{R} 0$ resection, postoperative complications, early mortality, and OS seem to be similar between the two groups. Therefore, LLR could be safely and feasibly performed for patients with multiple HCC meeting the Milan criteria compared to OLR. Further large sample size and well-designed trials should be conducted to validate our results.

\section{Ethical Approval}

This study was approved by the clinic trial Ethics Committee of West China hospital.

\section{Authors' Contributions}

Conception of the work: P.Y.F., L.B. and W.Y.G.; analyzed the data: P.Y.F. and L.F.; wrote the paper: P.Y.F. and X.H.W.; revised the paper: P.Y.F. and L.F.; contributed to the operations: P.Y.F., L.F., X.H.W., L.X., L.B. and W.Y.G.

\section{Disclosure Statement}

No competing financial interests exist.

\section{References}

1. Lim KC, Chow PKH, Allen JC, Siddiqui FJ, Chan ESY, Tan SB. Systematic review of outcomes of liver resection for early hepatocellular carcinoma within the Milan criteria. Br J Surg 2012;99:1622-1629.

2. Lin S, Hoffmann K, Schemmer P. Treatment of hepatocellular carcinoma: A systematic review. Liver Cancer 2012;1:144-158.

3. Jiang L, Yan L, Wen T, et al. Comparison of Outcomes of Hepatic Resection and Radiofrequency Ablation for Hepatocellular Carcinoma Patients with Multifocal Tumors Meeting the Barcelona-Clinic Liver Cancer Stage A Classification. J Am Coll Surg 2015;221:951-961.

4. Kim H, Ahn SW, Hong SK, et al. Survival benefit of liver resection for Barcelona Clinic Liver Cancer stage B hepatocellular carcinoma. Br J Surg 2017;104:1045-1052.

5. Yin L, Li H, Li AJ, et al. Partial hepatectomy vs. transcatheter arterial chemoembolization for resectable multiple hepatocellular carcinoma beyond Milan Criteria: A RCT. J Hepatol 2014;61:82-88.

6. Ho MC, Huang GT, Tsang YM, et al. Liver resection improves the survival of patients with multiple hepatocellular carcinomas. Ann Surg Oncol 2009;16:848-855.

7. Ishizawa T, Hasegawa K, Aoki T, et al. Neither multiple tumors nor portal hypertension are surgical contraindications for hepatocellular carcinoma. Gastroenterology 2008; 134:1908-1916.

8. Buell JF, Cherqui D, Geller DA, et al. The international position on laparoscopic liver surgery: The Louisville Statement, 2008. Ann Surg 2009;250:825-830.

9. Shehta A, Han HS, Yoon YS, Cho JY, Choi Y. Laparoscopic liver resection for hepatocellular carcinoma in cirrhotic patients: 10-year single-center experience. Surg Endosc 2016;30:638-648.

10. Lee W, Han HS, Yoon YS, et al. Comparison of laparoscopic liver resection for hepatocellular carcinoma located in the posterosuperior segments or anterolateral segments: A case-matched analysis. Surgery 2016;160:1219-1226.

11. Komatsu S, Brustia R, Goumard C, Perdigao F, Soubrane O, Scatton O. Laparoscopic versus open major hepatectomy for hepatocellular carcinoma: A matched pair analysis. Surg Endosc 2016;30:1965-1974.

12. Franken C, Lau B, Putchakayala K, DiFronzo LA. Comparison of short-term outcomes in laparoscopic vs open hepatectomy. JAMA Surg 2014;149:941-946.

13. Witowski J, Rubinkiewicz M, Mizera M, et al. Meta-analysis of short- and long-term outcomes after pure laparoscopic versus open liver surgery in hepatocellular carcinoma patients. Surg Endosc 2018.

14. Kwon Y, Han HS, Yoon YS, Cho JY. Are large hepatocellular carcinomas still a contraindication for laparoscopic liver resection? J Laparoendosc Adv Surg Tech A 2015;25:98-102. 
15. Kim SH, Kim KH, Kirchner VA, Lee SK. Pure laparoscopic right hepatectomy for giant hemangioma using anterior approach. Surg Endosc 2017;31:2338-2339.

16. Mbah NA, Brown RE, Bower MR, Scoggins CR, McMasters KM, Martin RC. Differences between bipolar compression and ultrasonic devices for parenchymal transection during laparoscopic liver resection. HPB (Oxford) 2012;14:126-131.

17. Robinson SM, Hui KY, Amer A, Manas DM, White SA. Laparoscopic Liver Resection: Is There a Learning Curve? Digest Surg 2012;29:62-69.

18. Yoon YS, Han HS, Cho JY, Yoon CJ, Kim JH. Laparoscopic approach for treatment of multiple hepatocellular carcinomas. Surg Endosc 2012;26:3133-3140.

19. Liu F, Wei Y, Li H, et al. LigaSure versus CUSA for parenchymal transection during laparoscopic hepatectomy in hepatocellular carcinoma patients with cirrhosis: A propensity score-matched analysis. Surg Endosc 2018;32:2454-2465.

20. Liu F, Zhang J, Lei C, Wei Y, Li B. Feasibility of laparoscopic major hepatectomy for hepatic paragonimiasis: Two case reports. Medicine (Baltimore) 2016;95:e4939.

21. Liu F, Xu H, Li Q, et al. Outcomes of pure laparoscopic Glissonian pedicle approach hepatectomy for hepatocellular carcinoma: A propensity score matching analysis. Surg Endosc 2019;33:1155-1166.

22. Mazzaferro V, Regalia E, Doci R, et al. Liver transplantation for the treatment of small hepatocellular carcinomas in patients with cirrhosis. N Engl J Med 1996;334:693-699.

23. Dindo D, Demartines N, Clavien PA. Classification of surgical complications: A new proposal with evaluation in a cohort of 6336 patients and results of a survey. Ann Surg 2004;240:205-213.

24. Balzan S, Belghiti J, Farges O, et al. The "50-50 criteria" on postoperative day 5: An accurate predictor of liver failure and death after hepatectomy. Ann Surg 2005;242: 824-828, Discussion 828-829.

25. Ishizawa T, Hasegawa K, Kokudo N, et al. Risk factors and management of ascites after liver resection to treat hepatocellular carcinoma. Arch Surg 2009;144:46-51.

26. Rahbari NN, Garden OJ, Padbury R, et al. Post-hepatectomy haemorrhage: A definition and grading by the International Study Group of Liver Surgery (ISGLS). HPB (Oxford) 2011; 13:528-535.

27. Koch M, Garden OJ, Padbury R, et al. Bile leakage after hepatobiliary and pancreatic surgery: A definition and grading of severity by the International Study Group of Liver Surgery. Surgery 2011;149:680-688.

28. Jiang L, Liao A, Wen T, Yan L, Li B, Yang J. Living donor liver transplantation or resection for Child-Pugh A hepatocellular carcinoma patients with multiple nodules meeting the Milan criteria. Transpl Int 2014;27:562-569.

29. Feng K, Yan J, Li X, et al. A randomized controlled trial of radiofrequency ablation and surgical resection in the treatment of small hepatocellular carcinoma. J Hepatol 2012;57:794-802.
30. Meguro M, Mizuguchi T, Kawamoto M, et al. Clinical comparison of laparoscopic and open liver resection after propensity matching selection. Surgery 2015;158:573-587.

31. Xu HW, Liu F, Li HY, Wei YG, Li B. Outcomes following laparoscopic versus open major hepatectomy for hepatocellular carcinoma in patients with cirrhosis: A propensity score-matched analysis. Surg Endosc 2018;32:712-719.

32. Stoot JH, van Dam RM, Busch OR, et al. The effect of a multimodal fast-track programme on outcomes in laparoscopic liver surgery: A multicentre pilot study. HPB (Oxford) 2009;11:140-144.

33. Wong-Lun-Hing EM, van Dam RM, van Breukelen GJ, et al. Randomized clinical trial of open versus laparoscopic left lateral hepatic sectionectomy within an enhanced recovery after surgery programme (ORANGE II study). Br J Surg 2017;104:525-535.

34. He F, Lin X, Xie F, Huang Y, Yuan R. The effect of enhanced recovery program for patients undergoing partial laparoscopic hepatectomy of liver cancer. Clin Transl Oncol 2015;17:694-701.

35. Chen MF, Tsai HP, Jeng LB, et al. Prognostic factors after resection for hepatocellular carcinoma in noncirrhotic livers: Univariate and multivariate analysis. World J Surg 2003;27:443-447.

36. Lau H, Fan ST, Ng IO, Wong J. Long term prognosis after hepatectomy for hepatocellular carcinoma: A survival analysis of 204 consecutive patients. Cancer 1998;83: 2302-2311.

37. Han HS, Shehta A, Ahn S, Yoon YS, Cho JY, Choi Y. Laparoscopic versus open liver resection for hepatocellular carcinoma: Case-matched study with propensity score matching. J Hepatol 2015;63:643-650.

Address correspondence to:

Bo $\mathrm{Li}, \mathrm{MD}, \mathrm{PhD}$

Department of Liver Surgery

Center of Liver Transplantation

West China Hospital of Sichuan University

No. 37 Guoxuegang

Chengdu 610041

China

E-mail: cdhxlibo168@163.com

Yonggang Wei, MD

Department of Liver Surgery Center of Liver Transplantation

West China Hospital of Sichuan University

No. 37 Guoxuegang

Chengdu 610041

China

E-mail: dr_wyg@163.com 